University of South Carolina

Scholar Commons

$5-2-2005$

\title{
Temperature-Dependent Asymmetry of the Nonlocal Spin- Injection Resistance: Evidence for Spin Nonconserving Interface Scattering
}

Samir Garzon

Igor Žutić

Richard A. Webb

University of South Carolina - Columbia, webbra@mailbox.sc.edu

Follow this and additional works at: https://scholarcommons.sc.edu/phys_facpub

Part of the Physics Commons

Publication Info

Published in Physical Review Letters, Volume 94, Issue 17, 2005, pages 176601-1-176601-4.

Garzon, S., Žutić, I., and Webb, R.A. (2005). Temperature-Dependent Asymmetry of the Nonlocal SpinInjection Resistance: Evidence for Spin Nonconserving Interface Scattering. Physical Review Letters, 94(17), 176601-1 - 176601-4. doi: 10.1103/PhysRevLett.94.176601

(C) 2005 The American Physical Society.

This Article is brought to you by the Physics and Astronomy, Department of at Scholar Commons. It has been accepted for inclusion in Faculty Publications by an authorized administrator of Scholar Commons. For more information, please contact digres@mailbox.sc.edu. 


\title{
Temperature-Dependent Asymmetry of the Nonlocal Spin-Injection Resistance: Evidence for Spin Nonconserving Interface Scattering
}

\author{
Samir Garzon, ${ }^{1}$ Igor Žutić, ${ }^{1,2}$ and Richard A. Webb ${ }^{1,3}$ \\ ${ }^{1}$ Department of Physics and Center for Superconductivity Research, University of Maryland, College Park, Maryland 20742, USA \\ ${ }^{2}$ Center for Computational Materials Science, Naval Research Laboratory, Washington D.C. 20375, USA \\ ${ }^{3}$ Department of Physics and USC NanoCenter, University of South Carolina, Columbia, South Carolina 29208, USA
}

(Received 30 August 2004; published 2 May 2005)

\begin{abstract}
We report nonlocal spin injection and detection experiments on mesoscopic $\mathrm{Co}-\mathrm{Al}_{2} \mathrm{O}_{3}-\mathrm{Cu}$ spin valves. We have observed a temperature-dependent asymmetry in the nonlocal resistance between parallel and antiparallel configurations of the magnetic injector and detector. This strongly supports the existence of a nonequilibrium resistance that depends on the relative orientation of the detector magnetization and the nonequilibrium magnetization in the normal metal providing evidence for increasing interface spin scattering with temperature.
\end{abstract}

DOI: 10.1103/PhysRevLett.94.176601

Spin injection from a magnetic material is accompanied by a nonequilibrium magnetization or spin accumulation near the interfacial region. The interest to study spin accumulation, one of the key elements in spintronic applications [1], is not limited to novel spin-based devices. Spin injection can also be used as a sensitive spectroscopic tool to study fundamental properties such as the pairing symmetry of unconventional superconductors [2,3], Skyrmion excitations in the quantum Hall regime [4,5], and spincharge separation in non-Fermi liquids [6,7]. While spin accumulation in metals was first demonstrated by Johnson and Silsbee [8], the basis for understanding spin injection and, more generally, spin-polarized transport, dates back to Mott [9]. He noted that the electrical current in ferromagnets could be expressed as the sum of two independent and unequal parts for two different spin projections implying that the current is spin polarized. This concept of a "twocurrent model" together with the spin-dependent scattering at the magnetic interfaces has been successfully used to explain giant magnetoresistance (GMR) and tunneling magnetoresistance (TMR), key elements in applications such as magnetic hard drives and nonvolatile magnetic random access memory [10,11].

A conventional picture for spin injection across the ferromagnet/nonmagnetic metal $(F / N)$ interfaces [12$14]$ is provided by noting that, in each metal, the spinflip scattering is typically much weaker than the momentum scattering. This leads to a mean free path (MFP) $l$ which is much shorter than the spin diffusion length (SDL) $\lambda$, the characteristic scale for the decay of spin accumulation at each side of the interface. Within the two-current model one can then define local spin-resolved electrochemical potentials $\mu_{\sigma}, \sigma=\uparrow, \downarrow$ for carriers with majority and minority spin (with magnetic moment parallel and antiparallel to the magnetization $\mathbf{M}$ in a ferromagnet). In the steady state an electrical current driven across a $F / N$ junction will lead to spin accumulation $\propto\left(\mu_{\uparrow}-\mu_{\downarrow}\right)$, which is the balance between spins added by the magnetization
PACS numbers: 72.25.Ba, 72.25.Hg, 72.25.Mk, 75.75.+a

current and spins removed by spin relaxation. In the absence of interfacial spin-flip scattering, the spin-resolved current $I_{\sigma}$ is conserved across the interface [13,14], and the contact resistance for each spin can be expressed as

$$
R_{\sigma}=\left(\mu_{\sigma}^{F}-\mu_{\sigma}^{N}\right) / e I_{\sigma},
$$

where the indices $F, N$ label the quantities in the corresponding region at each side of the contact and $e$ is the proton charge. $R_{\uparrow} \neq R_{\downarrow}$ can be inferred from the effect of exchange splitting in the $F$ region, leading to spindependent Fermi wave vectors, transmission coefficients, and density of states. Spin accumulation in the $N$ region can act as a source of spin electromotive force which produces a voltage $V \propto\left(\mu_{\uparrow}-\mu_{\downarrow}\right)$ measurable by adding another ferromagnet [15].

Using the $F_{1} / N / F_{2}$ geometry depicted in Fig. 1(a) in which $F_{1}$ represents the spin injector and $F_{2}$ the spin detector, we performed nonlocal measurements of spin injection over a wide temperature range. Charge current is driven between the leads $T_{1}$ and $N_{1}$ while the nonlocal voltage $V_{\mathrm{NL}}$ is measured between the leads $T_{2}$ and $N_{2}$ which, in the absence of nonequilibrium spin, is an equipotential region without a charge current flow such that $V_{\mathrm{NL}}=0$. As compared to local measurements (current

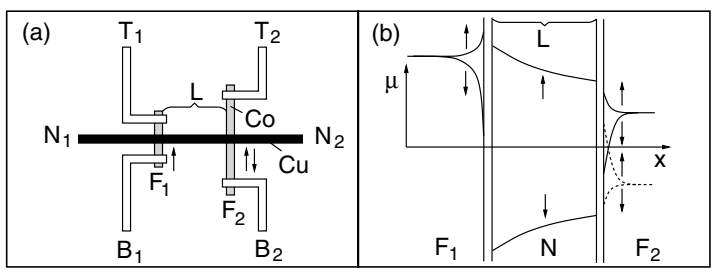

FIG. 1. (a) Sample geometry. Shaded (black) regions represent two Co ferromagnets ( $\mathrm{Cu}$ line). $T_{i}$ and $B_{i}$ are nonmagnetic measurement leads. (b) $\mu_{\sigma}$ for spin-up and spin-down electrons in the injector (left), normal metal (center), and detector (right) as a function of position. Detector $\mu_{\sigma}$ for parallel (solid) and antiparallel (dashed) magnetizations is shown. 
driven between $T_{1}$ and $T_{2}$ and voltage measured between $N_{1}$ and $N_{2}$ ), the nonlocal measurement has been shown to simplify the extraction of spurious effects (for example, anisotropic magnetoresistance and the Hall effect) from those intrinsic to spin injection [8,16]. In Fig. 1(b) we sketch a spatial profile of $\mu_{\sigma}$ where the presence of interfacial scattering leads to the discontinuity of the electrochemical potential $\mu=\left(\mu_{\uparrow}+\mu_{\downarrow}\right) / 2$ at each contact $[13,14,17]$.

For $F_{i} / N_{i}, i=1,2$ tunnel contacts (with resistance much larger than the characteristic values of the products $\rho_{N} \lambda_{N} / A_{N}$ and $\rho_{F} \lambda_{F} / A_{F}$ with $\rho$ the bulk resistivity and $A$ the cross-sectional area) conventional analysis gives a simple expression for the nonlocal resistance $[12,14,16]$

$$
R_{\mathrm{NL}}=\frac{V_{T_{2}-N_{2}}}{I_{T_{1}-N_{1}}}= \pm \frac{\rho_{N} \lambda_{N} \exp \left(-L / \lambda_{N}\right)}{2 A_{N}} P_{1} P_{2},
$$

where the signs " + " and "- " refer to the parallel $(\uparrow \uparrow)$ and antiparallel ( $\uparrow \downarrow)$ orientation of magnetizations in $F_{1}$ and $F_{2}$. $P_{i}$ is the single $F / N$ interface polarization of the current at contacts $F_{i} / N_{i}$ [18], which can be expressed as the ratio of spin-resolved contact resistances [19]

$$
P_{i}=\left(R_{i \uparrow}^{-1}-R_{i \downarrow}^{-1}\right) /\left(R_{i \uparrow}^{-1}+R_{i \downarrow}^{-1}\right) .
$$

Previous analysis of experimental data on spin injection in metallic systems has only considered the spin-valve effect due to the difference $R_{\mathrm{NL}}^{\Uparrow \uparrow}-R_{\mathrm{NL}}^{\uparrow \downarrow}$ between the nonlocal resistances for $\uparrow$ and $\uparrow \downarrow$ orientation of magnetizations in $F_{1}$ and $F_{2}[8,16,20,21]$. This approach was based on the understanding provided by either Eq. (2) or from a more general expression [14], not limited to the regime of tunnel contacts, which shows that the symmetric combination $R_{\mathrm{NL}}^{\Uparrow \downarrow}+R_{\mathrm{NL}}^{\uparrow \downarrow}$ vanishes identically.

In contrast, our findings suggest that both the symmetric and the antisymmetric combination of $R_{\mathrm{NL}}$,

$$
R_{S, A}=\left(R_{\mathrm{NL}}^{\Uparrow} \pm R_{\mathrm{NL}}^{\Uparrow \downarrow}\right) / 2,
$$

provide information about effects intrinsic to spin injection such as interfacial spin scattering. Our measurements of $R_{S}$ show that the usual assumption of $R_{\sigma}$ being equal for $\uparrow \uparrow$ and $\uparrow \downarrow$ orientation does not hold.

Our samples are electron beam defined $F / N / F$ structures with $\mathrm{Co}-\mathrm{Al}_{2} \mathrm{O}_{3}-\mathrm{Cu} F / N$ tunnel contacts as shown in Fig. 1(a). $36 \mathrm{~nm}$ of Co was thermally evaporated to form the magnetic injector and detector. An Ar ion mill was used to clean the magnetic contacts before the $2 \mathrm{~nm} \mathrm{Al}$ insulating level was thermally evaporated and oxidized for $4 \mathrm{~min}$ at pressures between 150 and 515 mTorr to obtain different contact resistances. Finally $54 \mathrm{~nm}$ of $\mathrm{Cu}$ was thermally evaporated to complete the $F / N$ contacts. The width of the $\mathrm{Cu}$ line was $100 \mathrm{~nm}$ while the injector and detector were $105 \mathrm{~nm}$ by $2.4 \mu \mathrm{m}$ and $95 \mathrm{~nm}$ by $4.5 \mu \mathrm{m}$, respectively, to ensure different coercivities allowing individual manipulation of their magnetization. The devices were measured at $4.2 \mathrm{~K}$ and between $77 \mathrm{~K}$ and room temperature [22]. Typical values of the SDL of $\mathrm{Cu}$ and
Co are much larger than the MFP, so the macroscopic diffusion equations are valid [14]. The contact resistance of both magnetic contacts was measured at $4.2 \mathrm{~K}$ and at room temperature, and found to be nearly temperature independent and between $9 \Omega$ and $1 \mathrm{k} \Omega$, depending on the oxidation parameters, so the approximation $R_{i} \gg$ $\rho \lambda / A$ is valid for the $F$ and $N$ regions.

ac currents between 10 and $50 \mu \mathrm{A}$ were injected from $T_{1}$ to $N_{1}$, see Fig. 1(a). Both the nonlocal voltage between $T_{2}$ and $N_{2}$, and the voltage at a $100 \mathrm{k} \Omega$ bias resistor were measured using lock-in amplifiers at $11 \mathrm{~Hz}$. First $R_{\mathrm{NL}}$ was measured at $4.2 \mathrm{~K}$ as the magnetic field parallel to the injector and detector $\left(B_{\|}\right)$was cycled between -300 and $300 \mathrm{mT}$ at $0.0003 \mathrm{~Hz}$. We observed the characteristic switching of the nonlocal resistance whenever the magnetic contacts became parallel or antiparallel [Fig. 2, Eq. (2)]. The magnitude of $R_{\mathrm{NL}}$ for $\uparrow \uparrow$ and $\uparrow \downarrow$ magnetic contacts is almost the same, $\left|R_{\mathrm{NL}}^{\Uparrow \uparrow}\right| \approx\left|R_{\mathrm{NL}}^{\Uparrow \downarrow}\right|$ so at low temperatures $R_{S}$ vanishes.

Hanle effect measurements [1] were performed to determine the degree of spin polarization and the spin diffusion length $\lambda_{N}$ of electrons in $\mathrm{Cu}$. The magnetic contacts were aligned by applying $B_{\|}$. After $B_{\|}$was turned off, an out-of-plane magnetic field $B_{\perp}$ was applied to induce spin precession as the electrons diffused from $F_{1}$ to $F_{2}$ with a time distribution $P(t)=1 / \sqrt{4 \pi D t} \exp \left(-L^{2} / 4 D t\right)$. Increasing $\left|B_{\perp}\right|$ also increases the precession angle of the electron spin making its projection onto $\mathbf{M}$ of $F_{2}$ smaller, hence decreasing $R_{\mathrm{NL}}^{\Uparrow}$. Diffusive motion of the electrons leads to a broad distribution of transport times and precession angles so a weighted average of the right-hand side of Eq. (2) must be used. The Hanle resistance, equivalent to the solution of Bloch-Torrey's equations [1,16,21], is

$$
R_{H}= \pm \frac{P_{1} P_{2} D \rho_{N}}{A_{N}} \int_{0}^{\infty} P(t) \cos (\omega t) e^{-D t / \lambda_{N}^{2}} d t
$$

where $\omega=g \mu_{B} B_{\perp} / \hbar$ is the Larmor frequency. The measured Hanle effect signal is weakly asymmetric (inset of Fig. 2) possibly because the two magnetic contacts were not perfectly parallel, or because the applied field had some

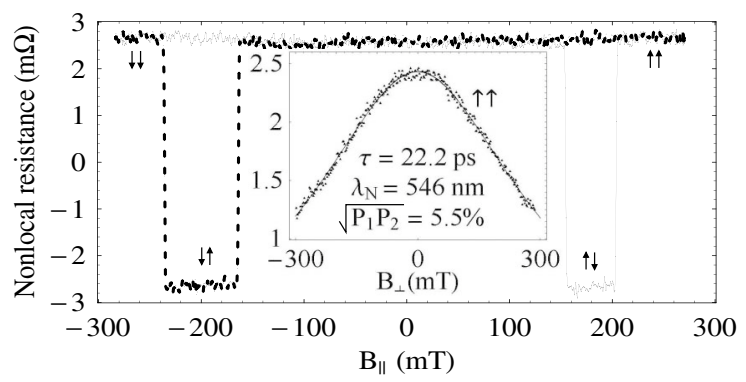

FIG. 2. $R_{\mathrm{NL}}$ as a function of parallel field at $4.2 \mathrm{~K}$ for an injector-detector separation of $430 \mathrm{~nm}$. The solid (dashed) trace is for increasing (decreasing) magnetic field. The arrows describe the direction of $\mathbf{M}$ of the injector and detector. Inset: Hanle effect for the parallel injector and detector demonstrating the precession of injected spins. 
nonzero in-plane component. Similar data was obtained for the case of an antiparallel injector and detector. By fitting the Hanle effect data to Eq. (5) we found that the spin diffusion length was $546 \mathrm{~nm}$ and the product of the spin polarizations of the two magnetic contacts was $\sqrt{P_{1} P_{2}}=$ $5.5 \%$. The value of the SDL is comparable to the values measured using GMR [23] $(450 \mathrm{~nm})$ and transparent $F / N / F$ spin valves [20] $(1000 \mathrm{~nm})$. The values of the spin polarization are also in agreement with those measured using $F / N / F$ spin valves [16].

We measured $R_{\mathrm{NL}}(T)$ for $100 \mathrm{~K}<T<300 \mathrm{~K}$ and observed a temperature-dependent asymmetry between $R_{\mathrm{NL}}^{\Uparrow \uparrow}$ and $R_{\mathrm{NL}}^{\uparrow \downarrow}$ as shown in Fig. 3(a). Our data show that $R_{S}=0$ only at low temperatures, but at room temperature $R_{S}$ is 3 times larger than the low temperature value of $R_{\mathrm{NL}}$. The temperature dependence of $R_{S}$ and $R_{A}$ is shown in Fig. 4(a) to illustrate that $R_{A}$ decreases linearly in this temperature range while the increase in $R_{S}$ is nonlinear. We see the expected [16,20] trend of decreasing $R_{A}$ as the temperature increases due to the increase in spin scattering in $\mathrm{Cu}$ and the reduction of the magnetization of Co due to magnons. For comparison we also measured $\rho(T)$ of $\mathrm{Co}$ and $\mathrm{Cu}$, which increased linearly for $100 \mathrm{~K}<T<300 \mathrm{~K}$ [Figs. 3(b) and 3(c)], while $R_{\mathrm{NL}}$ increased nonlinearly in that range [Fig. 3(a)]. Since $P_{1}$ should not change when $\mathbf{M}$ of the detector is reversed, the origin of $R_{S}$ can be traced to the difference in $P_{2}$ between $\uparrow \uparrow$ and $\uparrow \downarrow$ configurations [see Eq. (2)]. Hence the quantities $P_{S, A}=\left(P_{2}^{\Uparrow \uparrow} \pm P_{2}^{\Uparrow \downarrow}\right) / 2$ contain the relevant information of the spin transport across the $N / F_{2}$ interface. From previous studies [24] $P_{A}$ should have the same temperature dependence as the magnetization, $P(T)=P_{0}\left(1-\eta T^{3 / 2}\right)$. Since the momentum relaxation time $\tau_{p} \propto \tau$ (the spin relaxation time) where $\tau_{p} / \tau=a^{p}$ [1], we can use Fig. 3(c) to evaluate $\lambda_{N}(T)$. This, together with $P(T)$ and the measured value of the spin polarization from the Hanle effect $\left(P_{0}=5.5 \%\right)$, can be used to fit $R_{A}$ with Eqs. (2) and (4). Figure 4(b) shows the obtained $P_{A}$ together with a fit to $P(T)$ with $\eta=8.4 \times 10^{-5} \mathrm{~K}^{-3 / 2}$ and $a^{p}=6.6 \times 10^{-4}$. As expected from the interface effects $\eta$

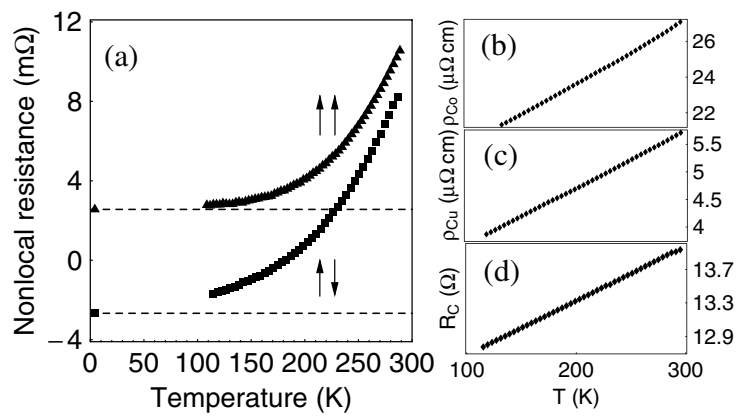

FIG. 3. (a) Temperature dependence of the nonlocal resistance for the parallel (triangles) and antiparallel (squares) injector and detector. Dashed lines represent $4.2 \mathrm{~K}$ values. (b),(c) Temperature dependence of the resistivity of $\mathrm{Co}$ and $\mathrm{Cu}$. (d) Typical temperature dependence of the contact resistance. is larger than the bulk value [24] and $a^{p}$ agrees very well with the previously measured value [20]. With the same two fitting parameters it is possible to extract the temperature dependence of $P_{S}$ from the measured $R_{S}$, as shown in Fig. 4(b), which can be fit well by a Fermi function that describes thermal activation with a characteristic temperature of $1227 \mathrm{~K}$. The measured offset $R_{S}$ can be modeled by assuming that the spin-dependent contact resistances depend on the relative alignment of the nonequilibrium magnetization in the normal metal and the magnetization in the ferromagnetic contact, so there are four independent contact resistances that describe the transport across each interface. This was shown to be possible in semiconductors due to nonlinear effects, but the argument is not valid in metals [25]. The most likely explanation for $R_{S} \neq 0$ is the existence of increasing spin-dependent scattering at the detector interface with temperature. In contrast with TMR, where the same charge current travels through an injector and detector, in the nonlocal geometry the absence of charge current at the detector justifies treating $F_{1}$ and $F_{2}$ differently. We confirmed this behavior by solving the diffusion equations including spin scattering at the $F_{2} / \mathrm{N}$ interface [26]. Equation (2) still holds if an effective spin polarization is redefined as $P_{2} \rightarrow \tilde{P}_{2}$, where [28]

$$
\tilde{P}_{2}=\frac{\left(R_{2 \uparrow}^{-1}-R_{2 \downarrow}^{-1}\right) \pm\left(R_{2 \uparrow}^{\prime-1}-R_{2 \downarrow}^{\prime-1}\right)}{\left(R_{2 \uparrow}^{-1}+R_{2 \downarrow}^{-1}\right)+\left(R_{2 \uparrow}^{\prime-1}+R_{2 \downarrow}^{\prime-1}\right)}=P_{A} \pm P_{S} .
$$

The primed quantities, absent in Eq. (3), represent the spin-scattering contact resistances [13] which allow the existence of nonvanishing $R_{S}$ and $P_{S}$ [29]. Even though $P_{A}$ includes spin-conserving and spin-scattering resistances, Fig. 4(b) shows clearly that it has the expected $1-\eta T^{3 / 2}$ dependence. This is clarified by Fig. 3(d) which shows that the measured contact resistance, proportional to the inverse of the denominator of Eq. (6), is weakly $T$ dependent for $100 \mathrm{~K}<T<300 \mathrm{~K}$. Therefore the strong $T$ dependence of $P_{S}$ comes from the difference in the interfacial spin scattering of the two spin channels at the detector $\left(R_{2 \uparrow}^{\prime-1}-R_{2 \downarrow}^{\prime-1}\right)$, which can be expected at a magnetic interface. While a detailed quantitative confirmation requires a fully microscopic picture, the increase of up to $30 \%$ in the relative scattering of spin $\uparrow$ and $\downarrow$ at $F_{2}$ for $100 \mathrm{~K}<T<300 \mathrm{~K}$ can be expected given the large spin polarization $(>50 \%)$ of surface states of Co [30].

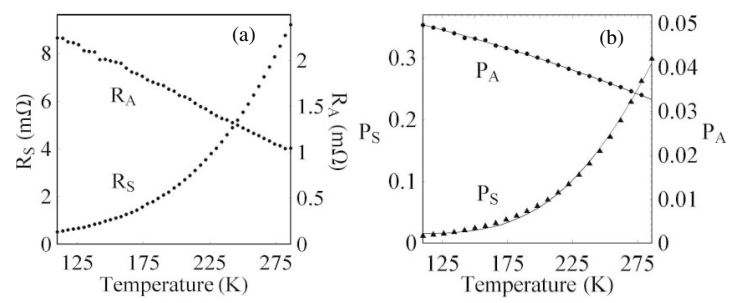

FIG. 4. Temperature dependence of the symmetric and antisymmetric components of (a) $R_{\mathrm{NL}}$, and (b) $P_{2}$ together with the fits from the proposed model. 
Figure 3 rules out spurious voltage offsets that can appear from the electrostatics of the cross geometry of the injector [31], since the value of that voltage would depend linearly on the contact resistance and the resistivity of both the $\mathrm{Cu}$ line and the Co injector, which are linear in temperature, as opposed to the measured offset which increases nonlinearly. Samples in which one or both of the Co contacts had been replaced by $\mathrm{Cu}$ showed $R_{S} \approx 0$, ruling out artifacts coming from the setup. Increasing the separation $L$ between $F_{1}$ and $F_{2}$ caused the value of $R_{S}$ to decrease and approach zero for large values of $L(\approx 2 \mu \mathrm{m})$, implying that $R_{S}$ is produced by the interplay of $F_{1}$ and $F_{2}$.

We verified these measurements by repeating them at dc, studied the response at higher frequencies, and replaced the lock-in amplifiers by a spectrum analyzer. We also checked the linearity of the signal with respect to the bias current. Leakage currents through the input impedance of the lock-in were negligible, and capacitive effects were ruled out by the dc measurements. All the results were consistent with those shown above. Heating, coupled with the difference in Seebeck coefficients of the two metals, can generate spurious voltages. However, these voltages should only appear at twice the fundamental frequency since the power goes as $I^{2}$. We studied this possibility by looking at the second harmonic response using both lock-in measurements and a spectrum analyzer, and were able to see a signal $\propto I^{2}$. Fur>thermore, we replaced Co by $\mathrm{Cr}$ whose relative Seebeck coefficient with respect to $\mathrm{Cu}$ is opposite in sign, and were able to see a change in the sign of the $I^{2}$ dependence, confirming the existence of thermal voltages at the second harmonic and not at the fundamental frequency.

In conclusion, temperature-dependent measurements of the nonlocal resistance in $F_{1} / N / F_{2}$ junctions reveal a previously overlooked asymmetry in the contact resistance for different relative orientations of the magnetizations in ferromagnets $F_{1}$ and $F_{2}$. We performed systematic control experiments to rule out a spurious origin of this asymmetry due to the electrostatic field distribution at imperfect contacts, Joule heating, and magnetothermal effects. Our analysis suggests that a conventional interpretation of spin injection which assumes spin-conserving interfaces needs to be generalized [32]. We developed a phenomenological model to show that the observed data is consistent with temperature-dependent spin-flip interfacial scattering. We believe that additional theoretical and experimental work will be required before a complete understanding of this new spin-flip effect is obtained.

This work was supported by NSF, NSA Laboratory for Physical Science, and NRC (I.Ž.). We thank M. Johnson, F. Jedema, and E. I. Rashba for useful discussions.

[1] I. Žutić, J. Fabian, and S. Das Sarma, Rev. Mod. Phys. 76, 323 (2004).

[2] V. A. Vas'ko et al., Phys. Rev. Lett. 78, 1134 (1997).
[3] J. Ngai et al., Appl. Phys. Lett. 84, 1907 (2004).

[4] A. H. MacDonald, Phys. Rev. Lett. 83, 3262 (1999).

[5] H. B. Chan et al., Phys. Rev. Lett. 83, 3258 (1999).

[6] Q. Si, Phys. Rev. Lett. 81, 3191 (1998).

[7] L. Balents and R. Egger, Phys. Rev. Lett. 85, 3464 (2000).

[8] M. Johnson and R. H. Silsbee, Phys. Rev. Lett. 55, 1790 (1985).

[9] N. F. Mott, Proc. R. Soc. A 153, 699 (1936).

[10] Spin Dependent Transport in Magnetic Nanostructures, edited by S. Maekawa and T. Shinjo (Taylor \& Francis, New York, 2002).

[11] S. S. P. Parkin et al., Proc. IEEE 91, 661 (2003).

[12] M. Johnson and R.H. Silsbee, Phys. Rev. B 35, 4959 (1987).

[13] E. I. Rashba, Eur. Phys. J. B 29, 513 (2002).

[14] S. Takahashi and S. Maekawa, Phys. Rev. B 67, 052409 (2003).

[15] R. H. Silsbee, Bull. Magn. Reson. 2, 284 (1980).

[16] F. J. Jedema et al., Nature (London) 416, 713 (2002).

[17] T. Valet and A. Fert, Phys. Rev. B 48, 7099 (1993).

[18] In contrast with TMR, $P_{2}$ should not be interpreted as the spin current polarization at the detector since in the nonlocal geometry no charge current reaches $F_{2}$ and therefore the spin current polarization at $F_{2}$ would be infinite. By definition $P_{2}$ is just the spin current polarization at $F_{2} / N$ when $F_{2}$ is used as an injector.

[19] B. T. Jonker et al., MRS Bull. 28, 740 (2003).

[20] F. J. Jedema, A.T. Filip, and B. J. van Wees, Nature (London) 410, 345 (2001).

[21] M. Johnson and R.H. Silsbee, Phys. Rev. B 37, 5326 (1988).

[22] The resistivity $\rho$ of the $\mathrm{Cu}$ line was $3.44 \mu \Omega \mathrm{cm}$ at $4.2 \mathrm{~K}$ and $5.76 \mu \Omega \mathrm{cm}$ at room temperature. Control samples for resistance determination of Co were fabricated under the same conditions as the spin-valve structures. Typical values for $\rho$ of Co were $18.7 \mu \Omega \mathrm{cm}$ at $4.2 \mathrm{~K}$ and $27.2 \mu \Omega \mathrm{cm}$ at room temperature. The corresponding mean free path and diffusion coefficient of $\mathrm{Cu}$ were $25.7 \mathrm{~nm}$ and $1.34 \times 10^{-2} \mathrm{~m}^{2} / \mathrm{s}$ at $4.2 \mathrm{~K}$ and $15.3 \mathrm{~nm}$ and $0.80 \times 10^{-2} \mathrm{~m}^{2} / \mathrm{s}$ at room temperature.

[23] Q. Yang et al., Phys. Rev. Lett. 72, 3274 (1994).

[24] C. H. Shang et al., Phys. Rev. B 58, R2917 (1998).

[25] I. Žutić, J. Fabian, and S. Das Sarma, Phys. Rev. Lett. 88, 066603 (2002).

[26] In contrast to [27] we have used a nonlocal geometry and nonzero spin-dependent contact resistances.

[27] M. Wawrzyniak and J. Barnas, Mater. Sci.-Poland 22, 537 (2004).

[28] As in Eq. (2), “+” and "-" refer to $\uparrow$ and $\uparrow \downarrow$ orientations of the magnetization of $F_{1}$ and $F_{2}$.

[29] The spin-resolved currents and electrochemical potentials in the presence of interface spin scattering are related by $I_{\uparrow}^{F}=\left(\mu_{\uparrow}^{N}-\mu_{\uparrow}^{F}\right) / e R_{\uparrow}+\left(\mu_{\downarrow}^{N}-\mu_{\uparrow}^{F}\right) / e R_{\downarrow}^{\prime}$ (and similarly for spin $\downarrow$ ) [13], which replace Eq. (1).

[30] S. N. Okuno, T. Kishi, and K. Tanaka, Phys. Rev. Lett. 88, 066803 (2002).

[31] M. Johnson, Ph.D. thesis, Cornell University, 1986.

[32] The efficiency and the temperature dependence of spin injection may likely be controlled by the spin-flip rate at the interface (a transport quantity) rather than, as it is usually assumed, by an estimated Curie temperature of an injector (a thermodynamic quantity). 